\title{
Managing a Two-Echelon Supply Chain under the Impact of Consumer Environmental Awareness and Carbon Labeling
}

\author{
Wei $\mathrm{Yu}^{1, \mathrm{a}}$ and Ruizhu Han ${ }^{1, \mathrm{~b}^{*}}$ \\ ${ }^{1}$ School of Economics and Management, Southeast University, Nanjing 211189, China \\ ayuweibill@126.com, bdaodao-777@163.com \\ the corresponding author
}

\begin{abstract}
Keywords: Carbon labeling; Consumer environmental awareness; Cost sharing
\end{abstract}
\begin{abstract}
This paper concentrates on the impact of consumer environmental awareness (CEA) on the decision behaviors of the supply chain members and the channel coordination strategies within a one-retailer-one-manufacturer supply chain under carbon labeling system. Based on the newsvendor model, this study compares two decision scenarios: the centralized and the decentralized models. The results suggest that the decision behaviors of the supply chain members are not only related to CEA and carbon labeling system, but also rely on the choice of the supply chain construction. Further, in order to achieve the same profit as the centralized model, we consider a cost-sharing contract to coordinate the decentralized supply chain. Finally, Sensitivity analyses are conducted to examine the impacts of changes in both CEA and carbon labeling on the performance of the supply chain.
\end{abstract}

\section{Introduction}

In recent years, the rise of CEA changes consumer purchasing behavior. Bemprad and Baranowski [1] point out that $51 \%$ of Americans are willing to pay more for the high environmental quality and $67 \%$ think it is very important to purchase products with environmental benefits on the basis of the BBMG Conscious Consumer Report. Many researchers are interested in studying the impact of CEA on supply chain operation[2-4]. But we concentrate on the supply chain members' low carbon promotion effort level and carbon emission level decisions.

Governments also adopt "carbon labeling" to switch consumer purchasing habits to low-carbon alternatives. Research has found that with the improvement of CEA, the consumers prefer to the low carbon products more and more, and are willing to pay more low carbon products [5]. The decision-makers should take the consumers concern on environment into consideration. $\mathrm{Xu}$ et al. [6] consider a two stage supply chain under cap-and-trade regulation and focus on the impact of carbon trade price on carbon labeling. The study finds that the increase of the carbon trade price can improve the optimal abatement level under certain conditions. Other researchers are interested in studying competition[7-9]. The above literature focuses solely on the single carbon emission reduction without considering any downstream firm's effort under carbon labeling system.

Under carbon labeling system, we consider a two-echelon supply chain consisting of one powerful manufacturer and one retailer and assume the demand is influenced by the retailer's low carbon promotion and manufacturer's carbon emission. Hence, this paper tries to investigate the following question:

1. How do both CEA and carbon labeling system influence the retailer's low carbon promotion and the manufacturer's carbon emissions comparing the centralized and decentralized contracts?

2. Under what conditions do the manufacturer and retailer decide to adopt the cooperative mechanism to coordinate the supply chain and earn more profits?

3. Whether the cost sharing strategy can effectively coordinate the manufacturer and the retailer and raise the profit of both sides?

Based on the above questions, our study compares two decision scenarios: the centralized model and the decentralized model. We derive the closed-from expressions of optimal carbon emission reduction 
level, low-carbon promotion level and retail price. Moreover, by introducing the carbon labeling system to the supply chain, the finding of the study will assist supply chain members to manage and optimize their carbon emission reduction, low-carbon promotion and pricing decisions.

This paper is organized as follows. The model and analysis are shown in section 2 . The supply chain coordination with wholesale price and the cost sharing contract are investigated in section 3 . Numerical examples are given in section 4 to illustrate some theoretical results obtained in previous sections. Section 5 is the conclusions and future research directions.

\section{Model and analysis}

In this paper, we consider a two-stage supply chain with a manufacturer and a retailer and assume only produce a green product that has three attributes, price, low-carbon promotion effort and carbon emission reduction level, influencing consumer demand.

According to [7] and [10] we can assume that the market demand $D$ is

$D=a-b p-k e+n$

To be specific, we summarize the model parameters and decision variables in Table 1.

Table 1 The description of the symbols

\begin{tabular}{|l|l|}
\hline Symbol & description \\
\hline Model parameters & \\
\hline$a$ & Initial market potential demand \\
\hline$b$ & Price sensitivity coefficient \\
\hline$k$ & Consumer environmental awareness (CEA) \\
\hline$\rho$ & Cost coefficient of carbon emission reduction \\
\hline$\theta$ & Cost coefficient of low-carbon promotion \\
\hline$c$ & Unit production cost \\
\hline$y$ & Initial unit amount of carbon emissions from production process \\
\hline Decision variables & \\
\hline$p$ & Unit retail price \\
\hline$w$ & Unit wholesale price \\
\hline$e$ & Carbon emission reduction level \\
\hline$n$ & Low-carbon promotion effort level of the retailer \\
\hline Other notations & \\
\hline$\pi_{I}$ & Supply chain profit in centralized model \\
\hline$\pi_{r}$ & Retailer's profit \\
\hline$\pi_{m}$ & manufacturer's profit \\
\hline Superscript $j$ & $\begin{array}{l}j \in\{c, d, c s\} \text { refers to centralized model, decentralized model and } \\
\text { cooperative model respectively }\end{array}$ \\
\hline
\end{tabular}

\section{Analysis of the centralized and the decentralized models.}

As a benchmark to evaluate coordination contract, we first formulate the centralized case (also termed as integrated supply chain). The total profit function can be model is

$\pi_{I}=(p-c)(a-b p-k e+n)-\frac{1}{2} \rho(y-e)^{2}-\frac{1}{2} \theta n^{2}$ 
To be specific, the cost of reducing per unit carbon emission to a level $e$ is $\frac{1}{2} \rho(y-e)^{2}$. We also assume the retailer's low carbon promotion effort level cost is $\frac{1}{2} \theta n^{2}$.

In the decentralized model, the manufacturer and the retailer make their profit maximization as their goal respectively. Hence, we describe their relationship as a Stackelberg game, in which the manufacturer is the Stackelberg leader. So the problem is formulated as follows:

$$
\begin{aligned}
& \pi_{r}=(p-w)(a-b p-k e+n)-\frac{1}{2} \theta n^{2} \\
& \pi_{m}=(w-c)(a-b p-k e+n)-\frac{1}{2} \rho(y-e)^{2}
\end{aligned}
$$

Solving both the centralzied and decentralized models, we derive the optimal solutions, which are summarized in Table2.

Table 2. Summary of the optimal solution under the centralized and decentralized model

\begin{tabular}{|c|c|}
\hline The centralized model & The decentralzied model \\
\hline$e^{c^{*}}=\frac{b \theta(c k+2 y \rho)-a k \theta-y \rho}{2 b \theta \rho-k^{2} \theta-\rho}$ & $e^{d^{*}}=\frac{a k \theta+2 y \rho-b \theta(c k+4 y \rho)}{k^{2} \theta+(2-4 b \theta) \rho}$ \\
\hline$p^{c^{*}}=\frac{(a-k y) \theta \rho-c\left(k^{2} \theta+\rho-b \theta \rho\right)}{2 b \theta \rho-k^{2} \theta-\rho}$ & $p^{d^{*}}=\frac{(a-k y) \rho-b^{2} c \theta \rho+b\left(-3(a-k y) \theta \rho+c\left(k^{2} \theta+\rho\right)\right)}{b\left(k^{2} \theta+(2-4 b \theta) \rho\right)}$ \\
\hline$n^{c^{*}}=\frac{(a-b c-k y) \rho}{2 b \theta \rho-k^{2} \theta-\rho}$ & $n^{d^{*}}=\frac{(a-b c-k y) \rho}{4 b \theta \rho-k^{2} \theta-2 \rho}$ \\
\hline$\pi_{I}^{*}=\frac{(a-b c-k y)^{2} \theta \rho}{2\left(2 b \theta \rho-k^{2} \theta-\rho\right)}$ & $\pi_{r}^{d^{*}}=\frac{(-a+b c+k y)^{2} \theta(2 b \theta-1) \rho^{2}}{2\left(k^{2} \theta+(2-4 b \theta) \rho\right)^{2}}$ \\
& $\pi_{m}^{d^{*}}=\frac{(a-b c-k y)^{2} \theta \rho}{8 b \theta \rho-2 k^{2} \theta-4 \rho}$ \\
\end{tabular}

According to above profit function, we have the following conclusion.

\section{Comparisons between two Types of Supply Chain Models.}

In this section, we compare the optimal decisions of carbon emission level, low carbon promotion effort level and retail price under the centralized and the decentralized models, respectively.

Proposition 1. For the manufacturer:

a) If $\left(0<y<y_{1}, 0<k<\frac{\sqrt{(2 b \theta-1) \rho}}{\sqrt{\theta}}\right)$, or $\left(y_{1}<y<y_{2}, 0<k<k_{1}^{d}\right)$, or $\left(y_{2}<y<y_{3}, 0<k<k_{1}^{c}\right)$, then $\left(\frac{\partial e^{c}}{\partial k}, \frac{\partial e^{d}}{\partial k}\right)<0$.

b) If $\left(y_{1}<y<y_{2}, k_{1}^{d}<k<\frac{\sqrt{(2 b \theta-1) \rho}}{\sqrt{\theta}}\right)$, then $\frac{\partial e^{c}}{\partial k}<0$ and $\frac{\partial e^{d}}{\partial k}>0$. 
c) If $\left(y_{2}<y<y_{3}, k_{1}^{c}<k<k_{1}^{d}\right)$, then $\frac{\partial e^{c}}{\partial k}>0$ and $\frac{\partial e^{d}}{\partial k}<0$.

d) If $\left(y_{2}<y<y_{3}, k_{1}^{d}<k<\frac{\sqrt{(2 b \theta-1) \rho}}{\sqrt{\theta}}\right)$, then $\left(\frac{\partial e^{c}}{\partial k}, \frac{\partial e^{d}}{\partial k}\right)>0$.

In which $y_{1}=\frac{(a-b c) \sqrt{\theta}}{\sqrt{2(2 b \theta-1) \rho}}, y_{2}=\frac{(a-b c) \sqrt{\theta}}{\sqrt{(2 b \theta-1) \rho}}, y_{3}=\frac{a-b c}{k}, k_{1}^{c}=\frac{y(2 b \theta-1) \rho-\sqrt{(2 b \theta-1) \rho\left(y^{2}(2 b \theta-1) \rho-(a-b c)^{2} \theta\right)}}{(a-b c) \theta}$ and $k_{1}^{d}=\frac{1}{(a-b c) \theta}\left[4 b y \theta \rho-2 y \rho-\sqrt{2} \sqrt{2 \rho^{2}(2 b \theta-1)^{2} y^{2}-(a-b c)^{2}(2 b \theta-1) \theta \rho}\right]$

Proposition 1 show that no matter in the centralized system or in the decentralized system, the manufacturer's carbon emission level $e$ is decreasing with CEA $k$ if the manufacturer's initial carbon emission $y$ is low; The manufacturer's carbon emission level $e$ is decreasing with CEA $k$ increasing in the centralized model, or the 's carbon emission level $e$ is increasing with CEA $k$ in the decentralized system if the manufacturer's initial carbon emission $y$ is moderate; Finally, no matter what kind of contracts the supply chain apply, the manufacturer's optimal carbon emission reduction level $e$ is increasing with CEA $k$ if the manufacturer's initial carbon emission level $y$ and CEA $k$ are high. The above results indicate that CEA can more effectively encourage the manufacturer to produce more less carbon intensive products if manufacturer's initial carbon emission satisfies the certain condition in centralized supply chain system. Next, for high levels of $y$, in order to reduce effectively carbon emission, smart both firms should adopt the integrated supply chain only if CEA is not relatively high.

Proposition 2. For the retailer:

a) If $\left(0<y<y_{2}, 0<k<k_{3}^{c}\right)$, or $\left(y_{2}<y<\frac{(a-b c)}{k}, 0<k<\frac{\sqrt{(2 b \theta-1) \rho}}{\sqrt{\theta}}\right)$, then $\left(\frac{\partial n^{c}}{\partial k}, \frac{\partial p^{c}}{\partial k}, \frac{\partial n^{d}}{\partial k}, \frac{\partial p^{d}}{\partial k}\right)<0$.

b) If $\left(0<y<y_{1}, k_{3}^{c}<k<k_{3}^{d}\right)$, or $\left(y_{1}<y<y_{2}, k_{3}^{c}<k<\frac{\sqrt{(2 b \theta-1) \rho}}{\sqrt{\theta}}\right)$, then $\left(\frac{\partial n^{c}}{\partial k}, \frac{\partial p^{c}}{\partial k}\right)>0$ and $\left(\frac{\partial n^{d}}{\partial k}, \frac{\partial p^{d}}{\partial k}\right)<0$

c) If $\left(0<y<y_{1}, k_{3}^{d}<k<\frac{\sqrt{(2 b \theta-1) \rho}}{\sqrt{\theta}}\right)$, then $\left(\frac{\partial n^{c}}{\partial k}, \frac{\partial p^{c}}{\partial k}, \frac{\partial n^{d}}{\partial k}, \frac{\partial p^{d}}{\partial k}\right)>0$.

In which $k_{3}^{c}=\frac{1}{y \theta}\left[a \theta-b c \theta-\sqrt{(a-b c)^{2} \theta^{2}-y^{2}(2 b \theta-1) \theta \rho}\right], k_{3}^{d}=\frac{1}{y \theta}\left[a \theta-b c \theta-\sqrt{(a-b c)^{2} \theta^{2}-2 y^{2}(2 b \theta-1) \theta \rho}\right]$

Proposition 2 indicates that no matter what kind of models the supply chain is, both the retailer's low carbon promotion effort level $n$ and retail price $p$ are increasing with the improvement of CEA $k$ if the manufacturer's initial carbon emission $y$ is low. However, both the retailer's low carbon promotion effort level $n$ and retail price $p$ are increasing with CEA $k$ increasing in the centralized model, or the consumers' low carbon promotion effort level $n$ and retail price $p$ are decreasing with CEA $k$ in the decentralized model if the manufacturer's initial carbon emission $y$ is moderate. Finally, no matter in the centralized model or in the decentralized model, the optimal retailer's low carbon promotion effort level $n$ and retail price $p$ are increasing with CEA $k$ if the manufacturer's initial carbon emission level $y$ is 
high. The implication of this result may be very interesting because the supply chain members should exert the centralized model in order to increase effectively the low carbon promotion effort level and retail price.

Form Proposition 1 and Proposition 2, we conclude that the improvement of CEA and carbon labeling are beneficial to the sustainable development of our society. Therefore, how to let more consumers develop the preference for eco-products and is an important matter to be considered. It is generally accepted that CEA can be affected by the publicity of both the government and the supply chain members. Consequently, it is necessary for both the government and the supply chain members to devote attention to enhance CEA.

That is to say, smart both parties should design a new contract that can effectively coordinate the supply chain.

Proposition 3. For a given initial carbon emission, (1) if $0<k<\sqrt{(2 b \theta-1) \rho} / \sqrt{\theta}$,

$$
\text { then } e^{c}<e^{d}, n^{c}>n^{d} \text { and } \pi^{c}>\pi_{m}^{d}+\pi_{r}^{d} ;(2)\left\{\begin{array}{l}
p^{c}<p^{d}, \text { if } 0<k<\sqrt{(b \theta-1) \rho} / \sqrt{\theta} \\
p^{c}>p^{d}, \text { if } \sqrt{(b \theta-1) \rho} / \sqrt{\theta}<k<\sqrt{(2 b \theta-1) \rho} / \sqrt{\theta}
\end{array}\right.
$$

Proposition 3(1) indicates that with $k$ falling in the range of $0<k<\sqrt{(2 b \theta-1) \rho} / \sqrt{\theta}$, it will be beneficial to increase the low carbon promotion effort level of the retailer while decrease the carbon emission level of the manufacturer in decentralized model. In this case, the market demand in decentralized model is large. Consumers are willing to buy more eco-friendly products, which will lead to the integrated supply chain members earn more profits.

Proposition 3(2) shows that if CEA $k$ is relatively small $(0<k<\sqrt{(b \theta-1) \rho} / \sqrt{\theta})$, the retailer price is lower in centralized model than that in decentralized model. On the contrary, with $k$ increasing and falling in the range of $\sqrt{(b \theta-1) \rho} / \sqrt{\theta}<k<\sqrt{(2 b \theta-1) \rho} / \sqrt{\theta}$, the retailer price is higher in centralized model than that in decentralized model. From the above analyses, we see that the degree of CEA is very important for both members in centralized model. If the supply chain members can increase CEA, it will be beneficial to the increase of the retailer profits.

\section{Coordination with a Cost-Sharing Contract}

From the perspective of supply chain management, we know that decentralized result in double marginalization. In this section, we propose a cost-sharing contract to coordinate the decentralized supply chain system under carbon labeling system, which encourages the supply chain members to share the costs of low carbon promotion effort and carbon emission reduction level into the decentralized supply chain system. In the cost sharing contract, the fraction of the low carbon promotion cost that the retailer pays is $\beta_{1}$, while the manufacturer needs to undertake $1-\beta_{1}$ portion of the cost in low carbon promotion effort. The fraction of the carbon emission reduction cost that the retailer offers is $\beta_{2}$, while the manufacturer needs to undertake $1-\beta_{2}$ portion of the cost in carbon emission reduction. In the proposed new model, the supply chain profits and costs are simultaneously determined and fairly shared. When we assume the wholesale price is $w=\beta_{3} p+\left(1-\beta_{3}\right) c$, then the profits of the retailer and the manufacturer are as follow:

$$
\begin{aligned}
& \pi_{r}^{c s}=\left(1-\beta_{3}\right)(p-c)(a-b p-k e+n)-\frac{1}{2} \beta_{1} \theta n^{2}-\frac{1}{2} \beta_{2} \rho(y-e)^{2} \\
& \pi_{m}^{c s}=\beta_{3}(p-c)(a-b p-k e+n)-\frac{1}{2}\left(1-\beta_{1}\right) \theta n^{2}-\frac{1}{2}\left(1-\beta_{2}\right) \rho(y-e)^{2}
\end{aligned}
$$

If the costs sharing of low carbon promotion and carbon emission, we can derive the optimal decisions as follows: 


$$
\begin{aligned}
& e^{c s^{*}}=\frac{t_{1}+t_{2}}{s_{1}} \\
& n^{c s^{*}}=\frac{t_{3}}{s_{2}} \\
& p^{c s^{*}}=\frac{t_{4}+t_{5}}{s_{3}} \\
& t_{1}=a k \theta\left(\beta_{1}-1\right)\left(\beta_{3}-1\right)^{2}-b c k \theta\left(\beta_{1}-1\right)\left(\beta_{3}-1\right)^{2} \\
& t_{2}=2 a b k \theta^{2} \beta_{1}^{2} \beta_{3}-2 b^{2} c k \theta^{2} \beta_{1}^{2} \beta_{3}+y \rho\left(\beta_{2}-1\right)\left(2 b \theta \beta_{1}+\beta_{3}-1\right)^{2} \\
& t_{3}=(a-b c-k y) \rho\left(\beta_{2}-1\right)\left(\beta_{3}-1\right)\left(2 b \theta \beta_{1}+\beta_{3}-1\right) \\
& t_{4}=2 b \beta_{1}^{2} \theta^{2}\left(\beta_{2} \rho(a+b c-k y)-\rho(a+b c-k y)+\beta_{3} c k^{2}\right)-\left(\beta_{3}-1\right)^{2} c\left(\rho-\beta_{2} \rho+\theta k^{2}\right) \\
& t_{5}=2 b \beta_{1}^{2} \theta^{2}\left(\beta_{2} \rho(a+b c-k y)-\rho(a+b c-k y)+\beta_{3} c k^{2}\right)-\left(\beta_{3}-1\right)^{2} c\left(\rho-\beta_{2} \rho+\theta k^{2}\right) \\
& s_{1}=k^{2} \theta\left(\beta_{1}-1\right)\left(\beta_{3}-1\right)^{2}+2 b k^{2} \theta^{2} \beta_{1}^{2} \beta_{3}+\rho\left(\beta_{2}-1\right)\left(2 b \theta \beta_{1}+\beta_{3}-1\right)^{2} \\
& s_{2}=\left(k^{2} \theta+\rho-\rho \beta_{2}\right)\left(\beta_{3}-1\right)^{2}-2 b \theta^{2} \beta_{1}^{2}\left(2 b \rho \beta_{2}+k^{2} \beta_{3}-2 b \rho\right)-\theta \beta_{1}\left(\beta_{3}-1\right)\left(4 b \rho \beta_{2}+k^{2} \beta_{3}-k^{2}-4 b \rho\right) \\
& s_{3}=2 b \beta_{1}^{2} \theta^{2}\left(2 b \beta_{2} \rho-2 b \rho+\beta_{3} k^{2}\right)+\left(\beta_{3}-1\right) \beta_{1} \theta\left(4 b \beta_{2} \rho-4 b \rho+\beta_{3} k^{2}-k^{2}\right)-\left(\beta_{3}-1\right)^{2}\left(\theta k^{2}+\rho-\beta_{2} \rho\right)
\end{aligned}
$$

Proposition 4. The supply chain can be effectively coordinated in the cost-sharing contract; and the profits shared by the retailer and the manufacturer, i.e., $\pi_{r}^{c s}=\left(1-\beta_{3}\right) \pi_{I}, \pi_{m}^{c s}=\beta_{3} \pi_{I}, \pi_{m}^{c s}>\pi_{m}^{d^{*}}$ and $\pi_{r}^{c s}>\pi_{r}^{d^{*}}$ if $\frac{2 b \theta \rho-k^{2} \theta-\rho}{4 b \theta \rho-k^{2} \theta-2 \rho}<\beta_{3}<\frac{k^{4} \theta^{2}+3 k^{2} \theta(1-2 b \theta) \rho+3(1-2 b \theta)^{2} \rho^{2}}{\left(4 b \theta \rho-k^{2} \theta-2 \rho\right)^{2}}, \beta_{3}=1-\beta_{2}, \beta_{1}=\beta_{2}$.

Proposition 4 shows that the total profit of the supply chain under the cost-sharing contract can is equal to the in the centralized supply chain system. In other words, this means that the perfect coordination cab be attained. Thus, we conclude that the cost-sharing contract cab be accepted by the manufacturer and retailer.

\section{Numerical example}

\section{The Joint Impacts of $k$ and $y$ on Carbon Emission Effort.}

To compare the optimal carbon emission effort level under different contracts, we assume that the parameters are: $a=100, b=5, c=2, k \in[0,2], \rho=1$ and $\theta=1$.

From Fig.1, we find that when initial carbon emission is low $(y=20)$, the manufacturer's carbon emission level decreases with CEA $k$ in both the centralized and the decentralized models. When initial carbon emission is moderate $(y=30)$, the manufacturer's carbon emission level firstly decreases and then increases with CEA $k$ in the decentralized model. However, the manufacturer's carbon emission level decreases with CEA $k$ in the centralized model. When initial carbon emission is high $(y=40)$, the manufacturer's carbon emission level firstly decreases and then increases with CEA $k$ in both the centralized and the decentralized models. It can be concluded that when initial carbon emission is relatively low, the manufacturer' incentive to reduce the carbon emission increases with CEA $k$ in both the centralized and decentralized models. Moreover, when initial carbon emission remains moderate, the manufacturer is willing to reduce the carbon emission increases with CEA $k$ only in the centralized model. 


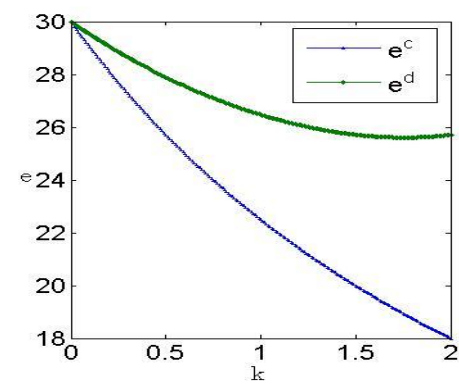

(1) $y=20$

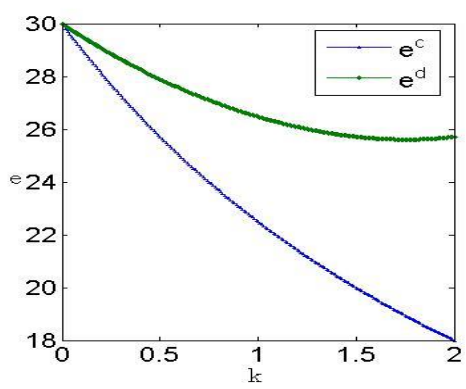

(2) $y=30$

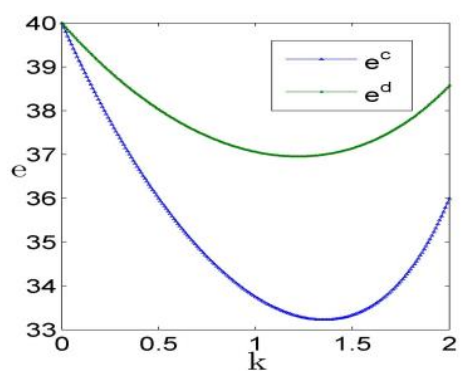

(3) $y=40$

Figure 1. The joint impacts of $k$ and $y$ on carbon emission effort

\section{The Joint Impacts of $k$ and $y$ on Low Carbon Promotion Effort level.}

To compare the optimal low carbon promotion effort level under different models, we assume that the parameters are: $a=100, b=5, c=2, k \in[0,2], \rho=1$ and $\theta=1$.

From Fig.2, we find that when initial carbon emission is low $(y=15)$, the retailer's low carbon promotion effort level increases with CEA $k$ in the centralized model and decreases with CEA $k$ in the decentralized model. When initial carbon emission is moderate $(y=25)$, the retailer's low carbon promotion effort level firstly decreases and then increases with CEA $k$ in the centralized model. However, the retailer's low carbon promotion effort level decreases with CEA $k$ in the decentralized model. When initial carbon emission is high $(y=35)$, the manufacturer's carbon emission level decreases with CEA $k$ in both the centralized and the decentralized models. It can be concluded that the higher green degree of the products (when initial carbon emission is low) has, the more effective the low-carbon promotion is in centralized model. Therefore, more consumers are more than willing to purchase more green products.

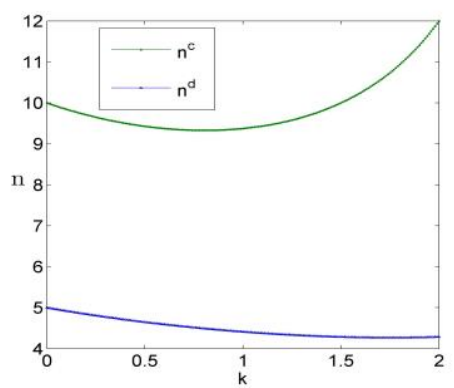

(1) $y=15$

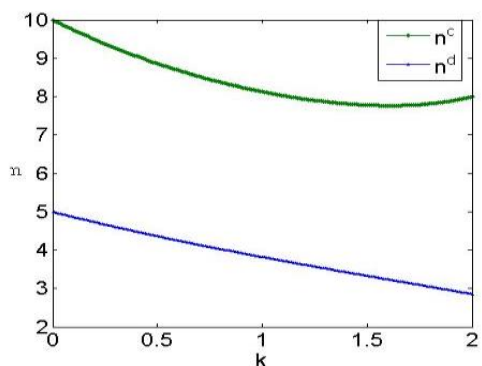

(2) $y=25$

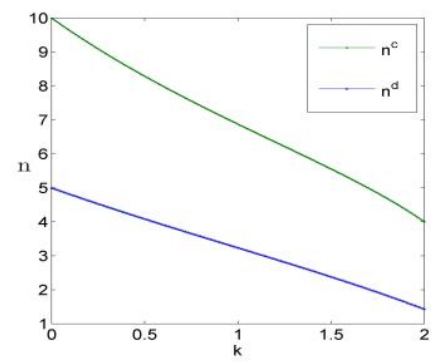

(3) $y=35$

Figure 2. The joint impacts of $k$ and $y$ on low carbon promotion effort level

\section{The Joint Impacts of $k$ and $y$ on Retail Price.}

From Fig.3, we find that regardless of which scenario initial carbon emission is, the retail price in centralized model is always lower than that in the decentralized model when $0<k<2$. But, when $k>2$, the retail price in centralized model is higher than that in the decentralized model. At the same time, it can be known that when initial carbon emission is low $(y=15)$, the retail price firstly decreases and then increases with CEA $k$ in both the centralized and the decentralized models. When initial carbon emission is moderate $(y=25)$, the retail price firstly decreases and then increases with CEA $k$ in the centralized model. However, the retail price decreases with CEA $k$ in the decentralized model. When initial carbon emission is high $(y=35)$, the retail price decreases with CEA $k$ in both the centralized 
and the decentralized models. It can be concluded that if government or the supply chain members can promote the consumers' low carbon preference, it will be beneficial to the increase of the retail price in the centralized model.

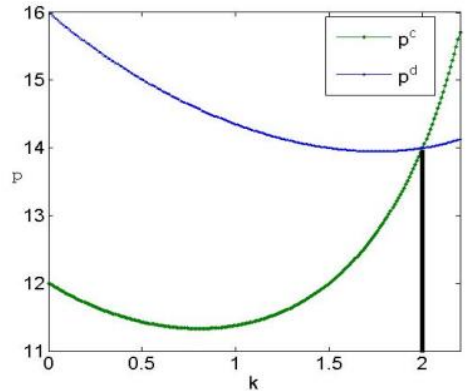

(1) $y=15$

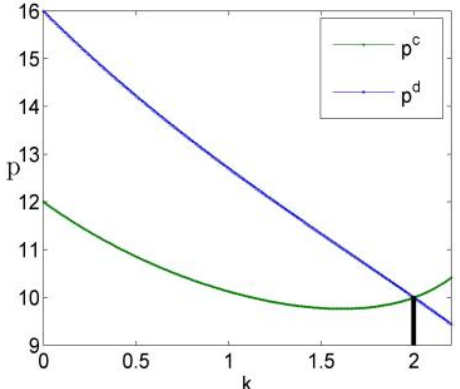

(2) $y=25$

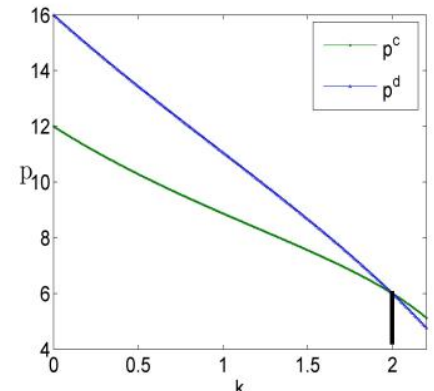

(3) $y=35$

Figure 3. The joint impacts of $k$ and $y$ on retail price

\section{Conclusions}

This paper incorporates the carbon labeling system and CEA into a two-echelon supply chain system. Based on the newsvendor model, this study compares two decision scenarios: the centralized and the decentralized models. The results suggest that the decision behaviors of the supply chain members are not only related to CEA and carbon labeling system, but also rely on the choice of the supply chain construction. By adopting the cost- sharing contract to coordinate the decentralized supply chain, both the retailer and the manufacturer can eliminate the double marginalization and implement 'win-win' situation.

In the future, we can extend the model to the online channel. Also, we can study dual channel supply chain with CEA and carbon labelling system, instead of the single channel supply chain in this research.

\section{Acknowledgments}

This work is supported by the National Natural Science Foundation of China under Grant (No. 71390335).

\section{References}

[1] R. Bemporad, and M. Baranowski, Conscious consumers are changing the rules of marketing. Are you ready? Highlights from the BBMG conscious consumer report. 2007, http://www.bbmg.com

[2] B. W. Jacobs, V. R. Singhal, and R. Subramanian, An empirical investigation of environmental performance and the market value of the firm, Journal of Operations Management, vol. 28 (2010) no.5, pp. 430-441.

[3] Z. K. Xiong, Z. Pan, and N. Guo, Impact of carbon tax and consumers' environmental awareness on carbon emissions in supply chains, Xitong Gongcheng Lilun Yu Shijian/system Engineering Theory \& Practice, vol. 34 (2014) no.9, pp. 2245-2252.

[4] L. Zhang, J. Wang, and J. You, Consumer environmental awareness and channel coordination with two substitutable products, European Journal of Operational Research, vol. 241 (2015) no.1, pp. 63-73.

[5] F. Adaman, N. Karalı, G. Kumbaroğlu, İlhan Or, B. Özkaynak, and Ünal Zenginobuz, What determines urban households' willingness to pay for co 2, emission reductions in turkey: a contingent valuation survey, Energy Policy, vol. 39 (2011) no.2, pp.689-698.

[6] X. Xu, P. He, H. Xu, and Q. Zhang, Supply chain coordination with green technology under cap-and-trade regulation, International Journal of Production Economics, (2016), pp.183.

[7] B. Yalabik, and R. J. Fairchild, Customer, regulatory, and competitive pressure as drivers of 
environmental innovation, International Journal of Production Economics, vo. 131 (2011) no.2, pp. 519-527.

[8] Z. Liu, T. D. Anderson, and J. M. Cruz, Consumer environmental awareness and competition in two-stage supply chains, European Journal of Operational Research, vo. 218 (2012) no.3, pp. $602-613$.

[9] K. Govindan, R. Khodaverdi, and A. Jafarian, A fuzzy multi criteria approach for measuring sustainability performance of a supplier based on triple bottom line approach, Journal of Cleaner Production, vol. 47 (2013) no.47, pp.345-354.

[10] H. Gurnani, and M. Erkoc, Supply contracts in -retailer interactions with -quality and retailer effort-induced demand, Naval Research Logistics, vol.55 (2008) no.3, pp. 200-217. 